\title{
Improving Liquid Crystal Alignment Ability and UV Sensitivity in Photodecomposition Polyimide Film
}

\author{
Hidenori Ishii ${ }^{1^{*}}$, Hisashi Tetsutani ${ }^{2}$, and Tatsuya $\mathrm{Nagi}^{3}$ \\ ${ }^{1}$ Display Materials Research Department, Materials Research Laboratories, \\ Nissan Chemical Corporation, \\ 488-6 Suzumi-cho, Funabashi-shi, Chiba 274-0052, Japan \\ 2 Analysis Research Department, Chemical Research Laboratories, \\ Nissan Chemical Corporation, \\ 2-10-1 Tsuboi-nishi, Funabashi-shi, Chiba 274-8507, Japan \\ ${ }^{3}$ Display Materials Research Team, R\&D Center, NCK Co., Ltd., \\ 127, Chupalsandan-ro, Paengseong-eup, Pyeongtaek-si, Gyeonggi-do, 17998, Korea \\ *ishiihi@nissanchem.co.jp
}

\begin{abstract}
A liquid crystal alignment film with fewer processes capable of imparting anisotropy was developed using a polyimide, which utilized the cleavage of a cyclobutane ring by irradiation of polarized UV light at $254 \mathrm{~nm}$. When an aromatic diamine with alkylene in the molecular center was used, an even methylene number of diamines showed superior liquid crystal alignment ability compared with diamines with an odd methylene number. Additionally, a diamine containing a molecular structure with a pre-imidized imide group adjoined to a cyclobutane ring was extremely effective in improving liquid crystal alignment ability and sensitivity to polarized UV light.
\end{abstract}

Keywords: FFS-mode, Photodecomposition polyimide, Liquid crystal alignment ability, UV sensitivity

\section{Introduction}

Liquid crystal displays (LCD) in smartphones, tablets and computers are not easily disarranged when pressed because many of them employ in-plane switching (IPS) and fringe-field switching (FFS) modes. Rubbing has been replaced by photoalignment as the method for treating alignment in liquid crystal alignment films used in LCD technology owing to the benefits of contrast and viewing angle. From the perspective of stability in backlight, and UV light when curing the sealant adhesive, we have been developing a photodecomposition polyimide capable of imparting anisotropy by irradiating polarized UV light at $254 \mathrm{~nm}$ [1-4]. If a process that grants anisotropy to the polyimide is shorter, then LCD manufacturing costs can be reduced. Therefore, a short-processed photodecomposition polyimide capable of imparting anisotropy has been developed by irradiating polarized UV light to cleave a cyclobutane ring with a specified direction after coating a substrate with polyamic acid solution; subsequently, it is pre-baked at approximately $80{ }^{\circ} \mathrm{C}$ to remove the solvent, and then post-baked at approximately $230{ }^{\circ} \mathrm{C}$ and imidized [5]. Additionally, when a solution of partially imidized polyamic acid-polyimide copolymer was used before coating the substrate during the same processes, it was confirmed that a superior liquid crystal alignment ability could be imparted with a small dosage of irradiated polarized UV light [6]. In this study, we investigated the relationship between the molecular structure derived from diamine and liquid crystal alignment ability. We also investigated in detail the relationship between the rate of imidization in a solution state, liquid crystal alignment ability, and UV sensitivity.

\section{Experimental}

2.1. Materials

Scheme 1 shows cyclobutane-1,2,3,4-tetra- 



Scheme 1. Synthesis of polyamic acids.
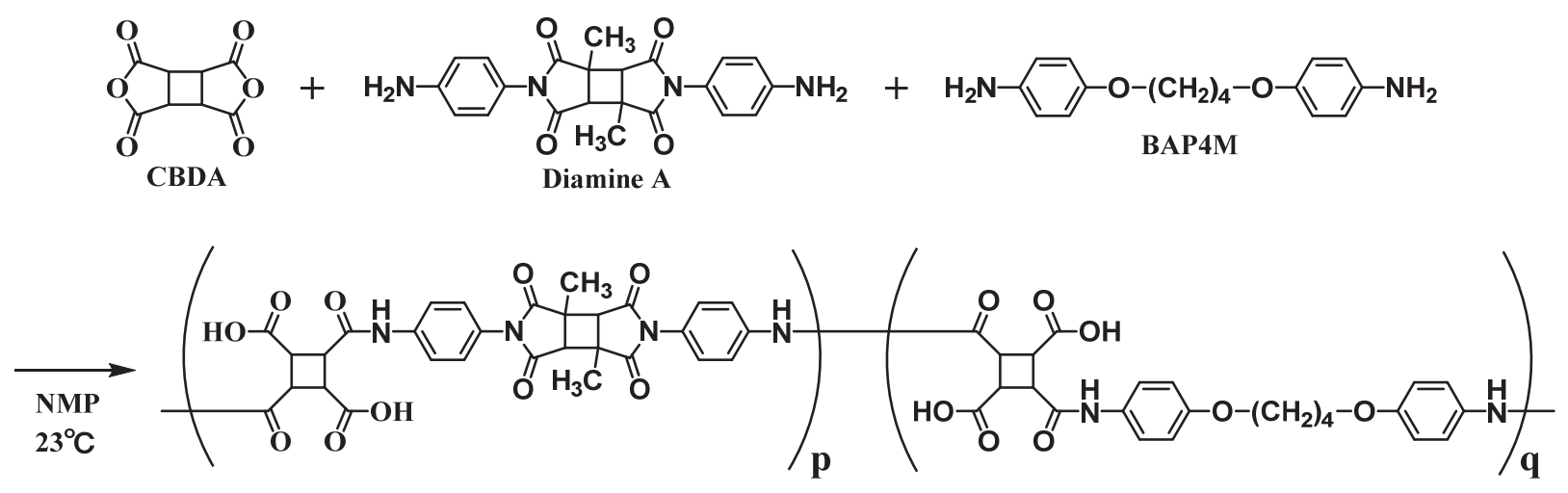

$p: q=10: 90,30: 70,40: 60,60: 40$

Scheme 2. Synthesis of polyamic acid-polyimide copolymers.

carboxylic dianhydride

1,4-diaminobenzene

1,2-bis(4-aminophenoxy)ethane

1,4-bis(4-aminophenoxy)butane

1,5-bis(4-aminophenoxy)pentane

1,6-bis(4-aminophenoxy)hexane

1,7-bis(4-aminophenoxy)heptane

1,9-bis(4-aminophenoxy)nonane

purchased as the raw materials required to

synthesize polyamic acid. Additionally, Scheme 2

(BAP4M),

(BAP5M),

(BAP6M),

(BAP7M), and

(BAP9M)
(CBDA), shows Diamine A, which was synthesized as the

(DB), raw material required to synthesize a polyamic

(BAP2M), acid-polyimide copolymer [6].

2.2. Synthesis of polyimide derivatives

Diamine was weighed in a four-necked flask equipped with a stirrer; subsequently, $\mathrm{N}$-methyl-2-pyrrolidone (NMP) was added and stirred to dissolve/disperse the diamine. The four-necked flask was immersed in a water bath 


\section{Polarization direction of UV light}

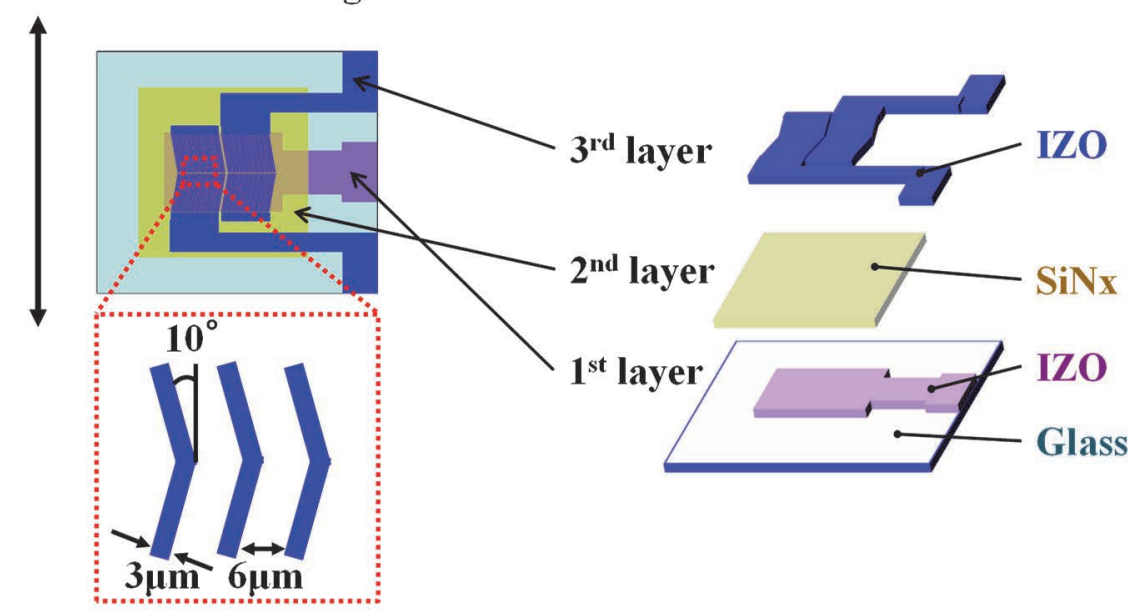

Fig. 1. FFS mode electrode-attached substrate.

and cooled, and CBDA was added while the solution was stirred. The leftover NMP was added to achieve a polymer concentration $10-12 \mathrm{wt} \%$, and the solution was stirred at $23{ }^{\circ} \mathrm{C}$ for $3-8 \mathrm{~h}$. When the total amount of diamine was $100 \mathrm{~mol} \%$, it was added to the solution to achieve a CBDA weight of 92-96 mol\% and adjusted to achieve a viscosity of $130-370 \mathrm{mPa} \cdot \mathrm{s}$ at a post-reaction solution temperature of $25{ }^{\circ} \mathrm{C}$. The obtained solution was fractionated in a conical flask containing a stirring rod and added in the order of NMP and a butyl cellosolve (BCS) to achieve a polymer concentration of $4.5-5 \mathrm{wt} \%$ and $\mathrm{BCS}$ amount of $30 \mathrm{wt} \%$ and stirred for $2 \mathrm{~h}$ at $23{ }^{\circ} \mathrm{C}$ to obtain a polymer solution for evaluating the alignment of the liquid crystals. Using the experimental procedure above, we obtained seven types of polyamic acid solutions (Scheme 1) and four types of polyamic acid-polyimide copolymer solutions (Scheme 2).

\subsection{Fabrication of liquid crystal cells}

After filtering the polyamic acid and polyamic acid-polyimide copolymer solutions through a 1.0 $\mu \mathrm{m}$ filter, they were deposited onto an FFS mode electrode-attached glass substrate via spin coating. Subsequently, they were pre-baked on a hot plate at $70{ }^{\circ} \mathrm{C}$ for $90 \mathrm{~s}$. Subsequently, polarized UV light was irradiated from a vertical direction onto the substrate using lithography equipment APL-L050121S1S-APW01 manufactured by Ushio Inc. through a 240LCF wavelength selector filter and a $254 \mathrm{~nm}$-type polarizer. The plane of polarization of the polarized UV light onto the substrate is inclined at $10^{\circ}$ from the top IZO electrode (3rd layer), as shown in Fig. 1. The exposure dose of polarized UV light was measured using the UVD-S254SB luminometer developed by Ushio Inc. and configured to the range 250 $1500 \mathrm{~mJ} / \mathrm{cm}^{2}$ at a wavelength of $254 \mathrm{~nm}$. Subsequently, the substrate was post-baked for 30 min using an infrared oven set at $230{ }^{\circ} \mathrm{C}$ to obtain a substrate with a 100 -nm-thick polyimide film with treated alignment. Additionally, to obtain a counter substrate, the same procedures above were applied to a glass substrate bearing a $4-\mu \mathrm{m}$-tall columnar spacer on which an ITO electrode was formed on the reverse side, resulting in a substrate with an alignment-treated polyimide film. These two polyimide film-attached substrates were paired to print a sealant with a shape that left behind a liquid crystal injection inlet on one side of a substrate with polyimide film. The sealant was then faced opposite to the polyimide film side of the other substrate so as to be parallel with the plane of polarization of the polarized UV light, where the two were pasted together and pressure bonded. Subsequently, the sealant was allowed to harden, thus producing an empty cell with a cell gap of $4 \mu \mathrm{m}$. The MLC-7026-100 liquid crystal (Negative LC manufactured by Merck \& Co., Inc.) was injected into the empty cell through a low-pressure injection method, and the injection inlet was sealed, obtaining an FFS-mode liquid crystal cell. Subsequently, the obtained FFS-mode liquid crystal cell was heated at $120{ }^{\circ} \mathrm{C}$ for $30 \mathrm{~min}$, 


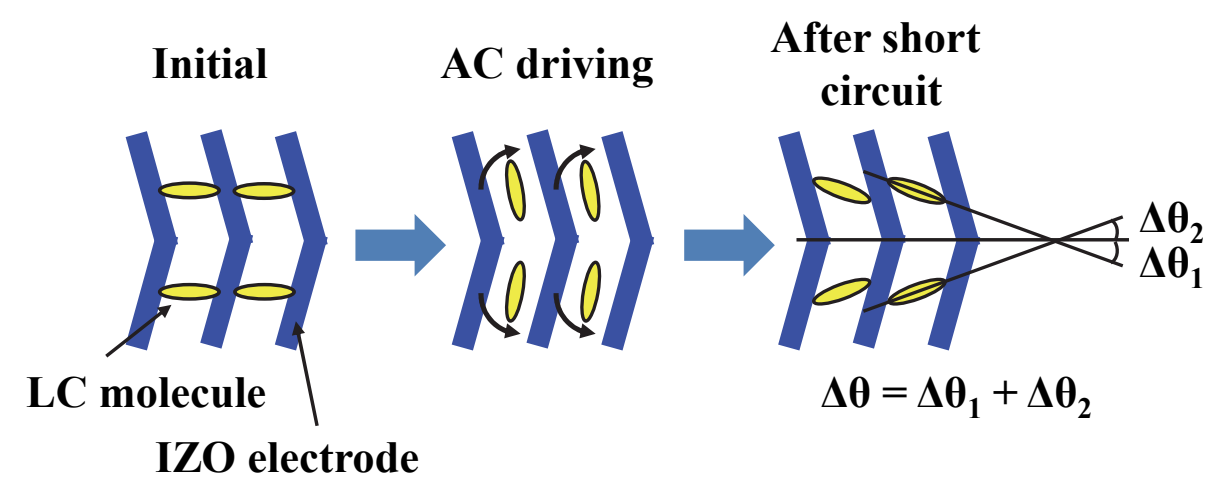

Fig. 2. Evaluation procedure of LC alignment ability.

left overnight at $23{ }^{\circ} \mathrm{C}$, and then used to evaluate the liquid crystal alignment ability.

\subsection{Evaluation of LC alignment ability}

Using the FFS-mode liquid crystal cell, an AC voltage of $16 \mathrm{Vpp}$ was applied for $96-168 \mathrm{~h}$ at a frequency of $30 \mathrm{~Hz}$ under a constant temperature of $70{ }^{\circ} \mathrm{C}$. Subsequently, the space between the top IZO electrode (3rd layer) and the bottom IZO electrode (1st layer) of the liquid crystal cell was short-circuited and left overnight at $23{ }^{\circ} \mathrm{C}$. Subsequently, the polarizer angle was measured by installing the liquid crystal cell between the two polarizers in a cross Nicols arrangement, and rotating the polarizer and analyzer while maintaining cross Nicols at each of the upper and lower regions of the curved electrode shown in Fig. 2. The amounts of change from the initial directional alignment of the liquid crystal's molecules, represented as $\Delta \theta_{1}$ and $\Delta \theta_{2}$, are calculated using the procedures above, and added together to obtain $\Delta \theta$ - the index for the liquid crystal alignment ability [7].

\section{Results and discussion}

Table 1 shows the results of the measurements of $\Delta \theta$, after a liquid crystal cell made from polyamic acid was AC-driven for $168 \mathrm{~h}$. When the exposure doses of polarized UV light were set to $600,900,1200$, and $1500 \mathrm{~mJ} / \mathrm{cm}^{2}$, the lowest values of $\Delta \theta$ were observed when exposed to the dosage of $1500 \mathrm{~mJ} / \mathrm{cm}^{2}$ across all samples, and the liquid crystal alignment ability was shown to be greater than that of liquid crystal cells manufactured with a small exposure dosage. When comparing BAP2M through to BAP9M, which possess an alkylene chain in the molecular structure derived from diamine, a molecular structure with an even methylene number produced a significantly smaller $\Delta \theta$ value than a molecular

Table 1. Results of $\Delta \theta$ of polyamic acids after AC driving for $168 \mathrm{~h}$.

\begin{tabular}{|c|c|c|c|c|c|}
\hline \multicolumn{2}{|c|}{ Polyamic acid } & \multicolumn{4}{|c|}{$\Delta \theta\left(^{\circ}\right)$} \\
\hline \multirow{2}{*}{$\begin{array}{c}\text { Tetracarboxylic } \\
\text { anhydride }\end{array}$} & \multirow{2}{*}{ Diamine } & \multicolumn{4}{|c|}{ Exposure dose of Polarized UV light $\left(\mathrm{mJ} / \mathrm{cm}^{2}\right)$} \\
\hline & & 600 & 900 & 1200 & 1500 \\
\hline CBDA & DB & 1.25 & 0.94 & 0.65 & 0.54 \\
\hline CBDA & BAP2M & 0.42 & 0.25 & 0.13 & 0.13 \\
\hline CBDA & BAP4M & 0.39 & 0.25 & 0.18 & 0.15 \\
\hline CBDA & ВАР5М & 0.94 & 0.62 & 0.54 & 0.51 \\
\hline CBDA & ВАР6М & 0.46 & 0.27 & 0.20 & 0.15 \\
\hline CBDA & BAP7M & 2.01 & 1.05 & 0.61 & 0.44 \\
\hline CBDA & ВАР9M & 1.34 & 0.75 & 0.51 & 0.37 \\
\hline
\end{tabular}


Table 2. Results of $\Delta \theta$ of polyamic acid-polyimide copolymers after AC driving for $96 \mathrm{~h}$.

\begin{tabular}{|c|c|c|c|c|c|c|c|c|c|c|}
\hline \multicolumn{3}{|c|}{ Polyamic acid-polyimide copolymer } & \multicolumn{8}{|c|}{$\Delta \theta\left(^{\circ}\right)$} \\
\hline \multirow{2}{*}{$\begin{array}{c}\text { Tetracarboxylic } \\
\text { anhydride }\end{array}$} & \multirow{2}{*}{$\begin{array}{c}\text { Diamine } \\
\text { Diamine A : BAP4M } \\
=\mathbf{p}: \mathbf{q}\end{array}$} & \multirow{2}{*}{$\begin{array}{l}\text { Imidization rate } \\
\text { in solution }(\%)\end{array}$} & \multicolumn{8}{|c|}{ Exposure dose of Polarized UV light $\left(\mathrm{mJ} / \mathrm{cm}^{2}\right)$} \\
\hline & & & 250 & 400 & 600 & 800 & 900 & 1000 & 1200 & 1500 \\
\hline CBDA & $0: 100$ & $\mathbf{0}$ & - & - & - & - & 0.21 & - & 0.16 & 0.13 \\
\hline CBDA & $10: 90$ & 9.7 & - & - & - & - & - & - & 0.20 & 0.16 \\
\hline CBDA & $30: 70$ & 24.6 & - & 0.08 & 0.11 & 0.15 & - & 0.20 & 0.22 & - \\
\hline CBDA & $40: 60$ & 30.3 & 0.03 & 0.03 & 0.07 & 0.24 & - & - & - & - \\
\hline CBDA & $60: 40$ & 39.5 & 0.04 & 0.02 & 0.10 & 1.55 & - & - & - & - \\
\hline
\end{tabular}

structure with an odd methylene number, confirming the even-odd effect. Factors related to the liquid crystal alignment ability of the liquid crystal alignment film in the IPS-mode LCD include viscoelastic behavior of the surface of the liquid crystal alignment film before and after $\mathrm{AC}$ driving, azimuthal anchoring energy, and uniaxial orientation of the polymer chain of the liquid crystal alignment film [7-11]. With these results alone, it is difficult to isolate the main factor that caused the even-odd effect. However, we inferred that the cause for the appearance of the even-odd effect in the $\Delta \theta$ value was that the principal polyimide chain was more likely to bend at least in the molecular structures of BAP2M through to BAP9M when the methylene number is odd, making the uniaxial orientation of the principal polyimide chain more likely to be disturbed.

Table 2 shows the results of the measurements of $\Delta \theta$, after a liquid crystal cell made from polyamic acid, or polyamic acid-polyimide copolymer was AC-driven for $96 \mathrm{~h}$. The rate of imidization in the solution state was calculated from the mole ratio of $\mathrm{CBDA}$ and Diamine $\mathrm{A}$. When observing the exposure-dose dependence of polarized UV light in $\Delta \theta$, the $\Delta \theta$ value is smallest for the samples $\mathrm{p}: \mathrm{q}=0: 100,10: 90$ under dosage of $1500 \mathrm{~mJ} / \mathrm{cm}^{2}$. However, the $\Delta \theta$ value is smallest for the samples $p: q=30: 70,40: 60,60: 40$ under dosage of $400 \mathrm{~mJ} / \mathrm{cm}^{2}$. From these results, it is considered that the sensitivity to polarized UV light is improved when the copolymerization ratio of Diamine $\mathrm{A}$ in the total amount of diamine is 30 mol\% or more. It is known that a cyclobutane ring derived from CBDA is easily cleaved by irradiated polarized UV light when the imidization ratio in the two adjoining imide groups is higher [12]. Additionally, in this experimental process, as the substrate was only heated up to $70{ }^{\circ} \mathrm{C}$ before being irradiated with polarized UV light after coating the substrate with a polyamic acid solution or polyamic acid-polyimide copolymer solution, it is considered that almost no imidization reaction would occur at this temperature. Therefore, we inferred that there is a threshold where UV light sensitivity is improved in this process in the range where the imidization rate is between $9.7 \%$ and $24.6 \%$ in the solution state. Additionally, the absolute value of $\Delta \theta$ is improved if the copolymerization ratio of Diamine $\mathrm{A}$ in the total amount of diamine is higher.

\section{Conclusion}

It is shown that an even methylene number in the molecular structures in BAP2M through to BAP9M resulted in a greater liquid crystal alignment ability. Additionally, increasing the copolymerization ratio of Diamine $\mathrm{A}$ in the total amount of diamine was effective in improving the liquid crystal alignment ability. Furthermore, a liquid crystal alignment film with excellent UV sensitivity was obtained when the copolymerization ratio of Diamine A was $30 \mathrm{~mol} \%$ or more.

\section{References}

1. M. Hasegawa and Y. Taira, J. Photochem. Sci. Technol., 8 (1995) 241.

2. J. L. West, X. Wang, Y. Ji, and J. R. Kelly, Dig. Tech. Pap.-Soc. Inf. Disp. Int. Symp., 26 (1995) 703.

3. X. Wang, D. Subacius, O. Lavrentovich, J. L. West, and Y. Reznikov, Dig. Tech. Pap.-Soc. Inf. Disp. Int. Symp., 27 (1996) 654.

4. J.-H. Kim, B. R. Acharya, S. Kumar, and K. R. Ha, Appl. Phys. Lett., 73 (1998) 3372.

5. T. Yamauchi, T. Matsuda, and N. Kondo, Japan 
Patent JP2013-235130A (2013).

6. S. H. Kwon, H. Han, J. H. Jo, H. A. Park, J. Y. Yoon, and H. S. Yun, Japan Patent JP2018-526670A (2018).

7. Y. Momoi, K. Tamai, K. Furuta, T. R. Lee, K. J. Kim, C. H. Oh, and T. Koda, J. Soc. Inf. Display, 18/2 (2010) 134.

8. M. Nishikawa, T. Kosa, and J. L. West, Jpn. J. Appl. Phys., 38 (1999) 334.

9. S. Hasegawa, Japan Patent JP2003-57147A
(2003).

10. S. Tanioka and J. Yokota, Japan Patent JP4599904B2 (2010).

11. Y. Matsui, N. Kunimatsu, and H. Sonoda, Japan Patent JP2010-72011A (2010).

12. S. Uchino, N. Kunimatsu, H. Sonoda, Y. Matsui, Y. Matsumoto, T. Yaguchi, H. Kaneko, and J. Tanaka, Japan Patent JP2011-107266A (2011). 